\section{An Evidential Approach to Map-Building for Autonomous Vehicles}

Daniel Pagac, Eduardo M. Nebot, and Hugh Durrant-Whyte

\begin{abstract}
In this work, we examine the problem of constructing and maintaining a map of an autonomous vehicle's environment for the purpose of navigation, using evidential reasoning. The inherent uncertainty in the origin of measurements of sensors demands a probabilistic approach to processing, or fusing, the new sensory information to build an accurate map.

In this paper, the map is based on a two-dimensional (2-D) occupancy grid. The sensor readings are "fused" into the map using the Dempster-Shafer inference rule. This evidential approach with its multivalued hypotheses allows quantitative analysis of the quality of the data. The map building system is experimentally evaluated using sonar data from real environments.
\end{abstract}

\section{INTRODUCTION}

The basic feature of an autonomous mobile robot is the capacity to operate independently in unknown or partially known environments. The term autonomous implies that the robot should be capable of reacting to static obstacles and unpredictable dynamic events which may impede the successful execution of a task. To achieve this level of robustness, processes need to be developed to provide solutions to localization, map-building, and navigation/obstacle avoidance problems. It could be argued that the map building task (updating obstacle locations relative to the AGV's position) and the localization problem (updating AGV's position relative to the environment) can be thought of as essentially a single process. However, in our view, these processes are quite distinct and independent since each may employ a separate set of sensors and processing algorithms. In this paper, we limit the scope to the problem of map building with particular reference to modeling of ultrasonic sensor information and probabilistic reasoning in map construction.

With a variety of sensor models, research is concentrated in two areas: the occupancy based map building (Elfes [3], Matthies and Elfes [7], and Lim and Cho [6]) and beacon recognition and tracking (Barshan and Kuc [1], Leonard and Durrant-Whyte [5], McKerrow [8]). The two approaches are, by no means mutually exclusive, in fact they supplement each other's weaknesses. The building of occupancy maps is well suited to path planning, navigation and obstacle avoidance because it explicitly models free space. However, occupancy maps are poor at localization (expensive algorithms are required, e.g., pattern matching). Consequently, beacon based methods have been successfully applied to the localization task. However, they fail to discern unknown types of obstacles or cluttered environments.

The Bayesian method has traditionally dominated the probabilistic sensor fusion in building occupancy maps. To quantify the sensor uncertainty, however, all conditional probabilities must be specified. Because of the difficulty of completing the model, the conditional probabilities are usually approximated. Such simplifications and poor original sensor model cause difficulties in building a reliable map. Also, the Bayesian theory requires $P(A)+P(\neg A)=1$, thus each

Manuscript received May 20, 1997. This paper was recommended for publication by Associate Editor A. Kusiak and Editor A. De Luca upon evaluation of the reviewers' comments.

The authors are with the Department of Mechanical and Mechatronic Engineering, The University of Sydney, Sydney NSW 2006, Australia (e-mail: pagac@mech.eng.usyd.edu.au; nebot@mech.eng.usyd.edu.au; hugh@mech.eng.usyd.edu.au).

Publisher Item Identifier S 1042-296X(98)06075-3.

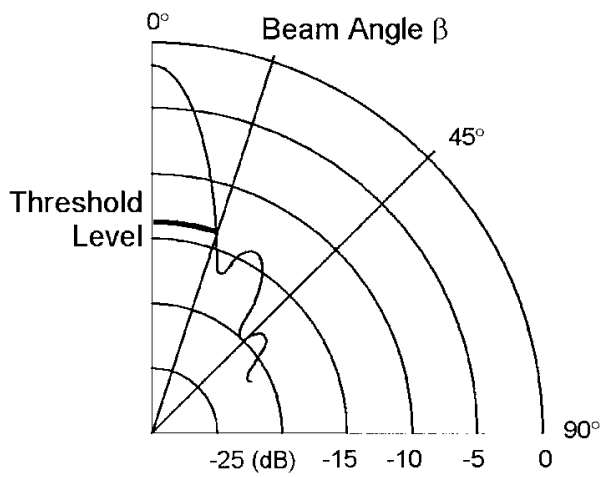

Fig. 1. A typical cross section of the beam pattern showing the threshold level.

cell in the map is initialized to $P_{i}$ (occupied $)=1-P_{i}$ (empty) $=0.5$ if no a priory information exists. This is the same as saying: "with $50 \%$ certainty, the cell $i$ is occupied," yet, no sensor readings have been collected. There is a general inability to quantify the amount and the quality of the information contained in the map when attempting to reduce the map to essentially a binary map as required for navigation. For example, with cell values close to uncertainty, such as $P_{i}($ occupied $)=0.55, P_{i}$ (empty $)=0.45$, one cannot deduce whether simply not enough information has been received, or whether the information received was contradictory thus making it likely that the particular cell $i$ will continue hovering close to the uncertainty value even with more readings from the given sensor. Such information, if it was available could be used to make decisions about the employment of different sensors and the validity of their data.

The Bayesian is certainly not the only approach that has been engaged to solve the problem of data fusion for this type of problem. Fuzzy maps have been applied to this problem with better results than Bayesian approach, as reported by Gambino et al. [12], as have more ad hoc methods such as histogram grids [2]. Also, the Dempster-Shafer formulation is not new to map-building and has long been considered as an alternative to the traditional Baysian approach. Tirumalai et al. have applied the theory to full three-dimensional (3-D) mapping of indoor environments employing stereo vision as the measuring sensor. This paper aims to show that using a straight-forward Dempster-Shafer approach with a more realistic sensor model a significant improvement in the accuracy of the map is achieved.

\section{SENSOR Model}

Map building using ultrasonic sensors has been addressed by many researchers and a substantial body of modeling and experimentation work has been presented by Elfes [3], Leonard [4], Kuc \& Siegel [5] and McKerrow [9]. The sonar's popularity can perhaps be attributed to it's availability, however, a number of it's characteristics have made it less attractive for map building. The most significant of these are the width of the beam, the specular properties of the environment and low bandwidth.

When a chirp, transmitted with a beam pattern shown in Fig. 1, causes an echo to be received some time later, it is difficult to ascertain what part of the beam has caused the return echo.

Clearly, if thresholding occurs, the only available evidence that can be ascertained from a single firing is the range to the obstacle and heading angle of the sensor. The only valid assumption that can be made is that the echo is generated somewhere on a arc at range $R$, within the sensor beam $\pm \beta$ (Fig. 2). 


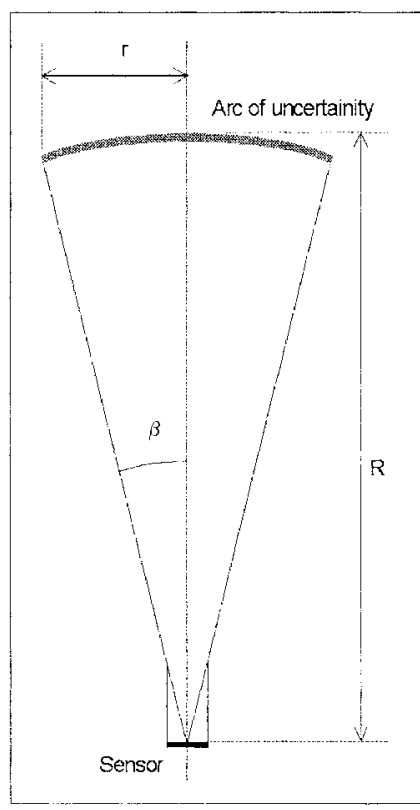

(a)

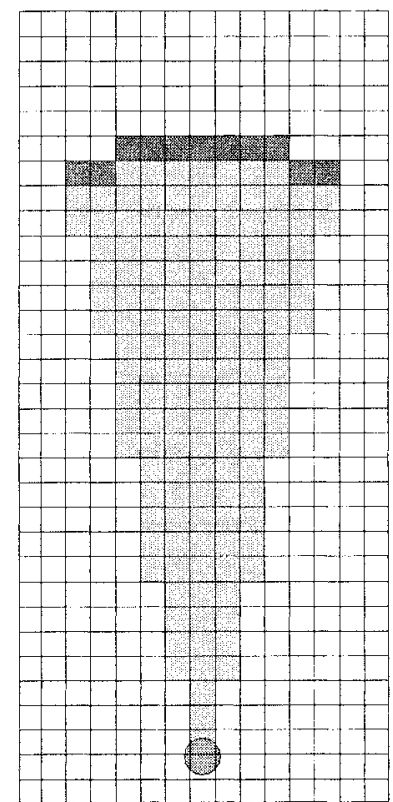

(b)
Fig. 2. (a) Sonar sensor with beam angle $\beta \approx 12^{\circ}$, showing the arc of uncertainty where the probability of obstacle occurring is equal, (b) projecting of the sensor beam on a rectangular grid assigns probabilities to cells of being empty, full, and ignorance outside the beam.

\section{A. Current Sensor Model and Bayesian Implementation}

To filter out the inherent uncertainties of the sensor the research has focused on probabilistic methods of evaluating the sensor confidence. Elfes [3], Matthies and Elfes [7], Borenstein and Koren [2], and Lim and Cho [6] all apply higher probabilities to the echo originating near the axis of the sensor and relatively low probabilities on either side. Since the introduction of this approach by Elfes it has been very popular, for example [6] and the application of Fuzzy Measures [12].

For the empty cells within the beam (the sector), the probability distribution is the greatest along the centre-line

$$
P(\theta, r)=P(\theta) P(r) \quad \text { for }-\beta \leq \theta \leq \beta, \quad 0 \leq r \leq R-\varepsilon
$$

and if the depth of the arc of uncertainty is less than cell side-length, the probability of cells along the arc being occupied is

$$
P(\theta, r)=P(\theta) \text { for }-\beta \leq \theta \leq \beta .
$$

The weight due to the angular distance from the centre is distributed according to the semicircular shape

$$
P(\theta)=1-\frac{\theta^{2}}{\beta^{2}} \text { for }-\beta \leq \theta \leq \beta
$$

and also, decaying the intensity with range (for the empty cells only)

$$
P(r)=1-\frac{r^{2}}{R^{2}} \quad \text { for } 0 \leq r \leq R-\varepsilon .
$$

Now, to represent the state by a single value, we normalize the probabilities around 0.5 with one denoting highest probability of cell being occupied and zero representing the maximum certainty that the cell is empty.

The resulting expression for modeling the cells in a sensor scan is

$$
P(\theta, r)\left\{\begin{array}{lll}
\frac{1-P(\theta) P(r)}{2} & \text { for }-\beta \leq \theta \leq \beta, \quad 0 \leq r \leq R-\varepsilon \\
1-\frac{P(\theta)}{2} & \text { for }-\beta \leq \theta \leq \beta, \quad r=R \\
\frac{1}{2} & \text { otherwise. }
\end{array}\right.
$$

The resulting probability distribution for a single scan is shown in Fig. 3.

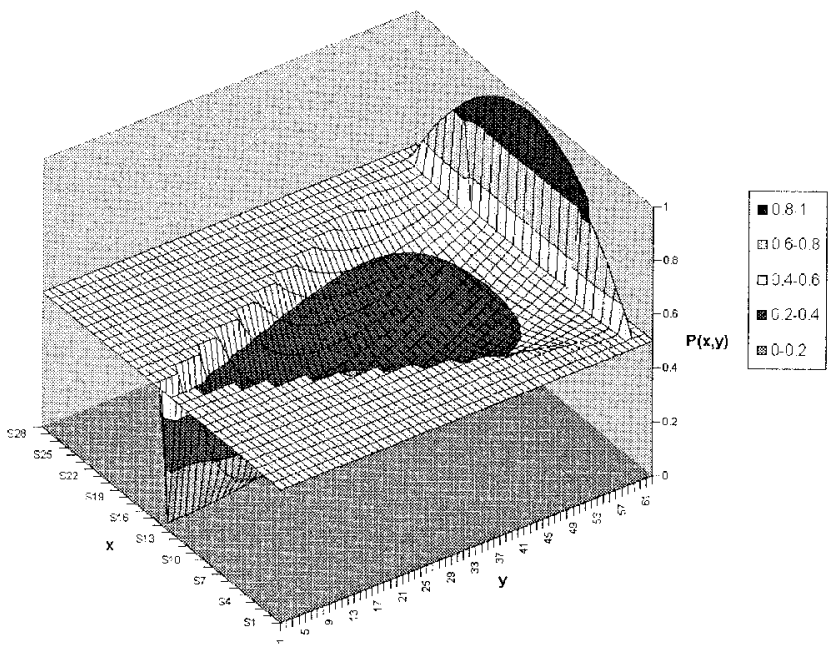

Fig. 3. Current model of the sonar sensor.

The scan data is combined using the Bayesian inference. The Bayesian formalism is represented using conditional probabilities, for example $P(A \mid B)$, which specify the belief in $A$ given event $B$ occurring.

$$
P(A \mid B)=\frac{P(B, A) P(A)}{P(B)} .
$$

So, if $P(A \mid B)=P(A)$, the piece of evidence $B$ has no effect on our certainty of $A$, and we say that the events $A$ and $B$ are independent. For derivation of Bayes' rule for combining probabilities the reader is directed to standard texts in statistics. Bayes' rule allows us to determine the conditional probability of the event $A$, given the observation of evidence $B$ using only knowledge about the probability of observing $B$ if $A$ has occurred and the prior probabilities $P(A)$ and $P(B)$.

The attraction of using Bayesian inference for map building stems from the property that the Bayes' updating rule facilitates recursive and incremental computational schemes, Pearl [33]. To illustrate this characteristic, let $x$ denote a hypothesis, $z_{n-1}=z^{1}, z^{2}, \cdots z^{n-1}$ denote a sequence of events observed in the past and $z^{n}$ denote a new observation. To calculate the new belief in $x, P\left(x \mid z_{n}, z^{n}\right)$ one could append the new observation to the past observation set and perform a global calculation on the whole set to arrive at the required value. To perform such operation would be time consuming and requires the complete history of observations. After a large number of observations, the calculation would become prohibitive for real-time operation. The calculation can be abridged by incremental updating. Once the probability $P\left(x \mid z_{n}\right)$ is known, the additional new evidence can be incorporated to obtain the posterior using

$$
P\left(x \mid z_{\text {in }-1}, z^{n}\right)=P\left(x \mid z_{\text {in }-1}\right) \frac{P\left(z^{n} \mid z_{\text {in }-1}, x\right)}{P\left(z^{n} \mid z_{\text {in }-1}\right)} .
$$

Another significant characteristic resulting from such computation is the property of associativity. This means that the order of the data stream does not change final result. It is an important result, because, in the case of autonomous vehicles, the order of the measurements depends on the path taken. So, although the order of the measurement might be different the evidence collected is nonetheless the same.

$P(\theta)$ and $P(r)$ distribute the probabilities according to the sensor model and the map being represented by occupancy values $P\left(x_{i} \mid\right.$ $z$ ) where the subscript $i$ denotes the cell and $z_{n}=z^{1}, z^{2}, z^{3}$, $\cdots, z_{n-1}, z^{n}$ denotes all readings including the new evidence $z^{n}$. 
So, the combination formula is

$$
P\left(x_{i} \mid z_{n}\right)=\frac{P\left(z^{n} \mid x_{i}\right) P\left(x_{i}\right)}{\sum_{j} P\left(z^{n} \mid x_{j}\right) P\left(x_{j}\right)} .
$$

This formulation was the starting point of our research. We endeavoured to improve the map-building process by analysing both, the sensor model and the fusion algorithm.

\section{B. New Sensor Model}

Applying the previous probability distributions over the sensor arc is fruitless if the input has been thresholded. Also, it might seem intuitive to decrease the probabilities of the empty cells as a function of range, however it is very difficult to justify a particular probability profile considering the sensor has to work in varied, unknown environments. We consider this departure from the current approach of modeling the sensor significant.

With the constraint that there is only one source of a sonar echo along the arc of uncertainty, the sum of probabilities of all the cells over the sensor arc being occupied (full, $F$ ) has to equal exactly 1

$$
\sum_{\forall \operatorname{cells}(i, j) \in \operatorname{Arc}} P_{F}(i, j)=1 \text {. }
$$

Furthermore, the arc of the sensor scan provides no information about the negation, $P_{E}(i, j)=P_{\neg F}(i, j)$, the cells being empty. Thus, for a continuous domain the probability mass distribution along the uncertainty arc would be

$$
P_{F}(i, j)=\frac{1}{2 R \beta} \quad \forall \operatorname{cells}(i, j) \in \operatorname{arc}
$$

where $R$ is the range and $\beta$ is the beam angle as shown in Fig. 2. By projecting the sensor beam onto a rectangular grid of discrete cells of equal size, with $n$ cell being affected by the sensor arc

$$
P_{F}(i, j)=\frac{1}{n} \quad \forall \operatorname{cells}(i, j) \in \operatorname{arc} .
$$

So, we assign equal probability distribution over the sensor arc, and since no evidence exists about the negation, $P_{E}(i, j)$, then we assign

$$
P_{E}(i, j)=0 \quad \forall \operatorname{cells}(i, j) \in \operatorname{arc} .
$$

Clearly, $P_{E}(i, j)+P_{F}(i, j) \neq 1$, so we diverge from the Bayesian approach and treat probabilities as evidence. By assigning $P_{E}(i, j)=$ 0 over the sensor arc, we propose that there no evidence to support the proposition that those cells are empty. Such modeling of ignorance leads directly to the theory of evidence.

Similarly, over the apparently unoccupied sector bounded by the two radii at $\pm \beta$ and the arc, there is no information about the probability of the cells being occupied

$$
P_{F}(i, j)=0 \quad \forall \operatorname{cells}(i, j) \in \text { sector. }
$$

This represents the lack of evidence about the cells within the sector being occupied, with the probability of being empty being constant over the whole sector

$$
P_{E}(i, j)=\rho \quad \forall \operatorname{cells}(i, j) \in \text { sector. }
$$

We believe this is the most accurate model of the sonar considering the return echo is thresholded as in the case of the commercial POLAROID sensor module.

Because of these properties, the ultrasonic range readings lend themselves well to the application of the Dempster-Shafer theory of evidence with the probabilities $P_{E}(i, j)$ and $P_{F}(i, j)$ corresponding to the basic probability assignments $m_{i, j}(E)$ and $m_{i, j}(F)$.

The practical implementation is quite simple in the map-building case and very similar to the Ming Vase Example described in the
Shafer's original text [10]. For a brief review of the Dempster-Shafer theory see Appendix A.

To build an occupancy map of the environment, we first construct a grid representing the whole space. Every discrete region of the map (each cell in the grid) is characterized by two states, empty and full. Thus, we define the field of discernment, $\Theta$, by the set

$$
\Theta=\{E, F\}
$$

where the $E$ and $F$ correspond to the possibilities that the cell is empty or full, respectively. The set of all subsets of $\Theta$ is the power set

$$
\Lambda=2^{\Theta}=\{\varnothing, E, F,\{E, F\}\} .
$$

The state of each cell is described by assigning basic probability numbers to each label in $\Lambda$. However, we know that for each cell $i, j$ in the grid

$$
m_{i, j}(\varnothing)=0
$$

and

$$
\begin{aligned}
\sum_{A \subset \Lambda} m_{i, j}(A)= & m_{i, j}(\varnothing)+m_{i, j}(E)+m_{i, j}(F) \\
& +m_{i, j}(\{E, F\})=1 .
\end{aligned}
$$

Considering this linear dependence and assuming $m_{i, j}(\varnothing)=0$, it is sufficient to store $m_{i, j}(E)$ and $m_{i, j}(F)$ only to fully represent the state of the system. Every cell in the map is first initialized, $m_{i, j}(E)=m_{i, j}(F)=0$ and $m_{i, j}(\{E, F\})=1$, representing total ignorance about the state of each cell. Then, as the AGV moves, scans of the environment are taken and fused into the map. The errors in the position of the AGV of less than the resolution of the map are absorbed by the cell size.

The basic probability assignment for the sensor arc, with $n$ cell being triggered are

$$
\left.\begin{array}{c}
m_{i, j}(F)=\frac{1}{n} \\
m_{i, j}(E)=0
\end{array}\right\} \quad \forall \operatorname{cells}(i, j) \in \operatorname{arc}
$$

within the beam

$$
\left.\begin{array}{l}
m_{i, j}(F)=0 \\
m_{i, j}(E)=\rho
\end{array}\right\} \quad \forall \operatorname{cells}(i, j) \in \text { sector }
$$

and outside the beam

$$
\left.\begin{array}{l}
m_{i, j}(F)=0 \\
m_{i, j}(E)=0
\end{array}\right\} \quad \forall \operatorname{cells}(i, j) \notin \text { arc, sector. }
$$

The basic probability mass assigned to the empty cells is constant and equal to the masses for the occupied cells on the arc $(1 / \rho)$. However, it is clear that large range readings will cause low basic probability assignments because $n$ will be relatively large. Finally, each cell in the map is updated using the Dempster's rule of combination

$$
m_{1} \oplus m_{2}(A)=\frac{\sum_{\forall B, C \in \Lambda: B \cap C=A} m_{1}(B) m_{2}(C)}{1-\sum_{\forall B, C \in \Lambda B \cap C=\emptyset} m_{1}(B) m_{2}(C)} .
$$

By adding subscripts $S$ and $M$ to the basic probability masses $m$, we describe the basic probability assignments of the sensor and the map. Explicitly, the new basic probability assignments for each cell in the map are

$$
\begin{aligned}
& m_{M} \oplus m_{S}(E)= \\
& \frac{m_{M}(E) m_{S}(E)+m_{M}(E) m_{S}(\{E, F\})+m_{M}(\{E, F\}) m_{S}(E)}{1-m_{M}(E) m_{S}(F)-m_{M}(F) m_{S}(E)}
\end{aligned}
$$




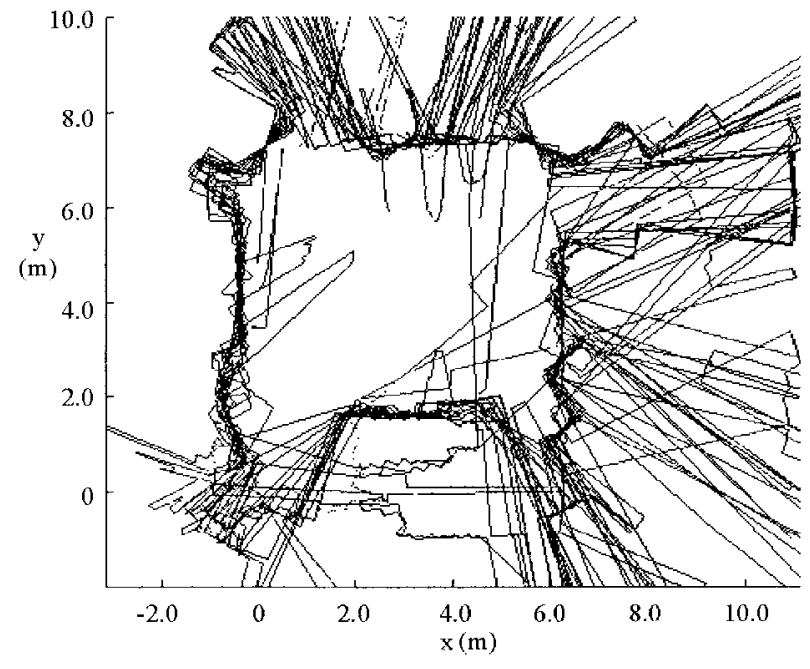

Fig. 5. Complete $360^{\circ}$ scans (in global coordinates) from 40 different positions in the space.

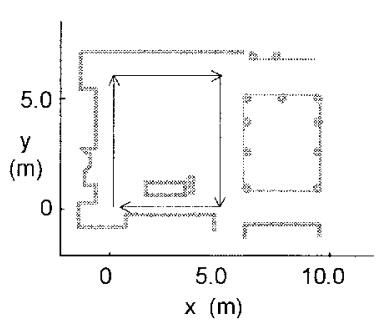

(a)

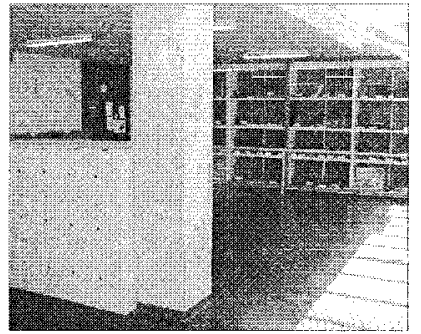

(b)
Fig. 6. (a) The path that generated the sequence of data for the example in Figs. 7 and 8, showing the position of the camera and (b) the photo of the environment (note glazed north wall).

\section{EXPERIMENTAL RESULTS}

We have built a ring of Polaroid ultrasonic transducers and a single sensor scanner with a sensor mounted on the shaft of a stepper motor. These transducers operate at $49 \mathrm{kHz}$, and project an axisymmetric beam with an included angle of approximately $22^{\circ}$.

Two large areas were surveyed out to a $200 \mathrm{~mm}$ grid with accuracy better than $3 \mathrm{~mm}$. Then, by placing the rotating sensor rig at each of the surveyed points, a set of 400 readings were taken and saved. To perform the mapping, a virtual path (a walk-through) was generated by retrieving the corresponding sonar scans sequentially and performing the fusion process. Other inputs included the resolution of the map, the initial robot position and orientation, the number of scans taken from each position and the maximum range. Due to the low bandwidth of the sonar and the computation order of the algorithm, these variables directly control the computation time.

The following example demonstrates the quality of building algorithm. Fig. 6 shows the path along which scans were taken at $200 \mathrm{~mm}$ intervals.

This particular run was done in an area constructed of a number of different types of materials, such as glass, brick and concrete walls, railings, doors, and is furnished with lockers, chairs, and water coolers. The cell size was $50 \mathrm{~mm}$, maximum range $10 \mathrm{~m}$, map size $200 \times 200$ cells (covering area of $10 \times 10 \mathrm{~m}^{2}$ ).

The final map is shown in Fig. 7, in a 3-D representation, and in Fig. 8 as grey level image. It can be clearly seen that most of the features of the environment (corners, walls, etc.) are accurately mapped. The difficulties of using the ultrasonic sensor in complex areas such as long specular walls are to large extent overcome by this method.

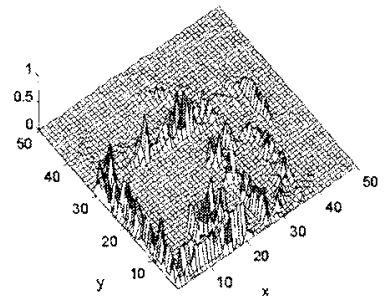

(a)

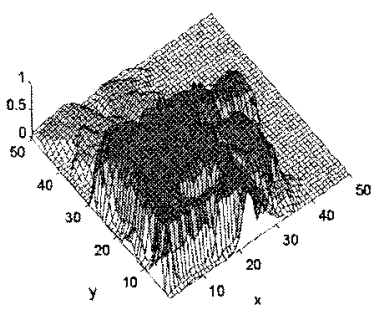

(b)
Fig. 7. Occupancy maps generated using 120 sonar scans showing the basic probability assignments (a) $m_{F, i, j}$ and (b) $m_{E, i, j}$. (Coordinates are in pixels, with each pixel representing area $200 \times 200 \mathrm{~mm}$, a patch of $4 \times 4$ cells. The reduction in resolution is due to clarity of presentation).

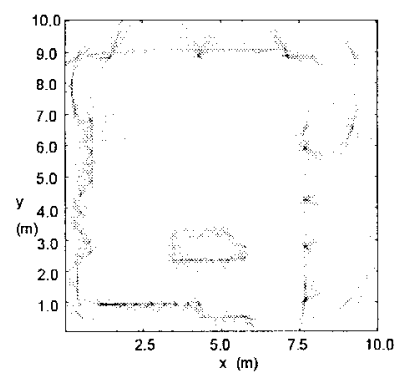

(a)

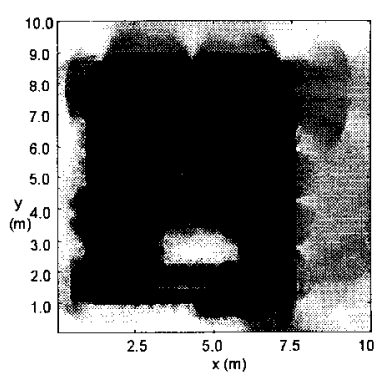

(b)
Fig. 8. Grey level images showing the basic probability assignments (a) $m_{F, i, j}$ and (b) $m_{E, i, j}$. (black: $m=1$, white: $m=0$ ).

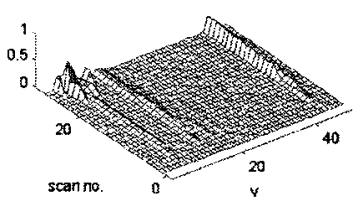

(a)

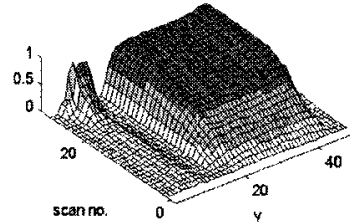

(b)
Fig. 9. Cross section of the map (corresponding to $x=18$ in Fig. 7), showing the accumulation of evidence; how the true states of the cells are evolving with time. The obstacle in the southern part of the room $(y \approx 12)$ is clearly defined.
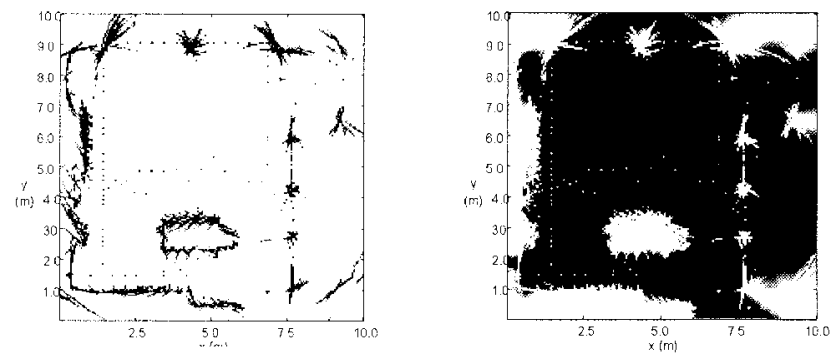

Fig. 10. Occupancy maps generated using the Bayes' fusion rule, values above and below 0.5 separated into full and empty map res. and normalized into the interval $\left[\begin{array}{ll}0 & 1\end{array}\right]$ for comparison to the D-S method.

To show the accumulation of evidence in particular cells, a cross section of the map is presented in Fig. 9. The basic probability assignments for $E$ and $F$ are plotted against time (360 scans).

Evaluating the occupancy maps, it is immediately apparent that the Dempster-Shafer approach provides much better solution to the map-building problem. The Bayes' method gives good results in certain cases, but its performance is generally very poor when the maps are compared to the Dempster-Shafer result Fig. 10. Certainly, 

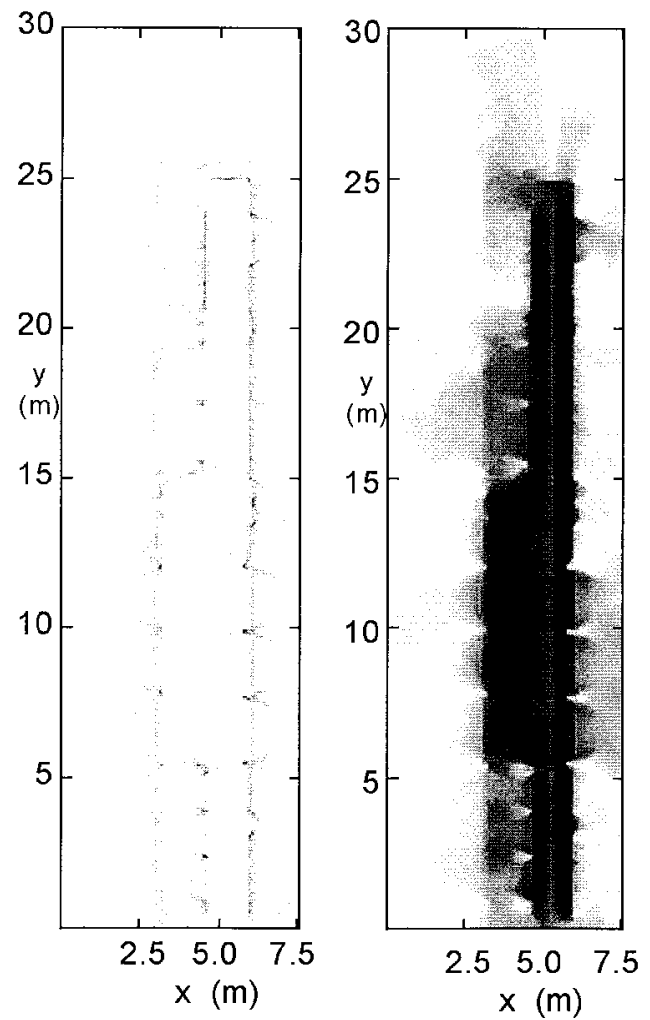

Fig. 11. Map of a corridor: (a) $m_{F, i, j}$ and (b) $m_{E, i, j}$.

diffusion into free-space still occurs (this highly apparent in all representations at the glazed wall on the northern boundary), however, in the Dempster-Shafer method this leakage is minimized but more importantly, a row of occupied cells clearly defines the barrier. This barrier is either not defined at all or very faint in the other representations. Fig. 8 clearly shows that in the D-S map there in fact are two glass walls at $y \approx 9 \mathrm{~m}$, between $y \approx(1.5,4.2) \mathrm{m}$ and $y \approx(4.4,7.1) \mathrm{m}$. Compare this to the result of the old method. This is certainly an exiting result considering the properties of the sensor and the environment.

The algorithm was further validated using data from a long corridor (max. $2.5 \mathrm{~m}$ wide and $24 \mathrm{~m}$ long) with very difficult surfaces such as long glazed walls and smooth concrete (Fig. 12). The cell size is again $50 \mathrm{~mm}$ and the virtual path executed start at coordinates $(5.2,24.5)$ and ends at $(5.2,0.5)$. With a range of building materials forming the environment the maps clearly show the obstacles as well as free areas (Fig. 11).

\section{CONCLUSION}

This work presents a novel application of the theory of evidence and significantly improves the map building process for autonomous vehicles. It also shows the importance of defining an accurate sensor model. The work considers the uncertainties of the ultrasonic sensor measurements and make use of the Dempster-Shafer reasoning to integrate sensor and modeling information. When compared with other probabilistic methods, the success of this method is attributed to the following characteristics of this method.

It differs from the Bayes approach by allowing support for more than one proposition at a time, rather than a single hypothesis. It is interval based, as defined by the upper and lower probability bounds, Pls and $\mathrm{Bel}$, allowing lack of data (ignorance) to be modeled adequately. This model no longer requires full description of

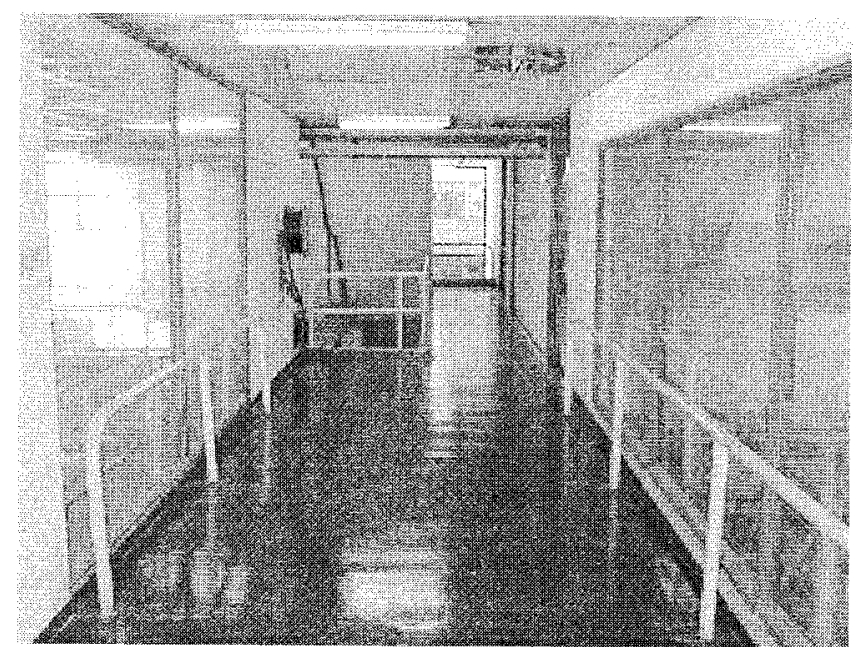

Fig. 12. Photo of the corridor.

conditional (or prior) probabilities and small incremental evidence can be adequately incorporated. Also, it allows to quantify the undistributed probability masses, thus making assessment about the quality of the posterior probabilities. The structure of 2-D map is largely independent of the method and can be implemented by other representations (grid, quadtrees, hextrees).

Experimental results with the ultrasonic sensor confirm the capabilities of a grid-based application and algorithms developed. It is evident that the Bayes approach is overly sensitive to outliers as also observed by Gambino et al. [12]. Whereas in [12] it is aimed to solve the problem using fuzzy logic and fuzzy measures with comparison to the benchmark Bayes approach, we have aimed to improve the sensor mode and employ the theory of evidence to fuse the measurements. It would certainly be interesting and to formally and objectively compare the results of the two approaches, fuzzy methods and the evidential reasoning.

\section{APPENDIX \\ DEMPSTER-SHAFER THEORY OF EVIDENCE}

The Dempster-Shafer theory of evidence is characterized by a frame of discernment (FOD), basic probability assignment (bpa), belief $(\mathrm{Bel})$ and plausibility $(\mathrm{Pls})$ functions and the Dempster's rule of combination (Shafer [10]).

The frame of discernment, denoted $\Theta$, is defined to be a finite set of labels representing mutually exhaustive events.

The basic probability assignment is the function $m: \Psi \rightarrow[0,1]$, where $\Psi$ is the set of all subsets of $\Theta$, the power set of $\Theta, \Psi=2^{\Theta}$. The function $m$ can be interpreted as distributing probabilities to each of the labels in $\Psi$, with the following criteria satisfied

$$
\begin{aligned}
\sum_{A \subset \Psi} m(A) & =1 \\
m(\varnothing) & =0 .
\end{aligned}
$$

Thus, the label $A$ is assigned a basic probability number $m(A)$ describing the degree of belief that is committed to exactly $A$. However, the total evidence that is attributed to $A$ is the sum of all probability numbers assigned to $A$ and its subsets

$$
\operatorname{Bel}(A)=\sum_{\forall B: B \subseteq A} m(B)
$$


The function $\mathrm{Bel}: \Psi \rightarrow[0,1]$ is the quantity of evidence supporting the proposition $A$ and has the following properties:

$$
\begin{aligned}
\operatorname{Bel}(\varnothing) & =0 \\
\operatorname{Bel}(\Theta) & =1 \\
\operatorname{Bel}(A)+\operatorname{Bel}(\neg A) & \leq 1 \\
\operatorname{Bel}(A) & \leq \operatorname{Bel}(B) \quad \text { if } A \subset B \\
\operatorname{Bel}(A \cap B) & =\min (\operatorname{Bel}(A), \operatorname{Bel}(B)) .
\end{aligned}
$$

The plausibility of a proposition $A$ can be thought of as the amount evidence that does not support its negation $\neg A$. It is defined as Pls $: \Psi \rightarrow[0,1]$, with the following properties:

$$
\begin{aligned}
P l s(A) & =1-\operatorname{Bel}(\neg A)=1-\sum_{\forall B: A \not \subset B} m(B) \\
P l s(A)-\operatorname{Bel}(A) & \geq 0 \\
P l s(A \cup B) & =\max (\operatorname{Pls}(A), P l s(B))
\end{aligned}
$$

The state of each label (described by bpa) is updated by combining a new independent source of evidence using the Dempster's rule of combination

$$
\begin{aligned}
& m_{1} \oplus m_{2}(A)=\frac{\sum_{\forall B, C \in \Psi: B \cap C=A} m_{1}(B) m_{2}(C)}{1-\sum_{\forall B, C \in \Psi B \cap C=\emptyset} m_{1}(B) m_{2}(C)} \\
& m_{1} \oplus m_{2}(\varnothing)=0 .
\end{aligned}
$$

The $\mathrm{Bel}$ and $\mathrm{Pls}$ functions are often denoted as upper and lower probabilities. The amount $P l s .(A)-\operatorname{Bel}(A)$ the additional undistributed (plausible) evidence that is compatible with both hypotheses $A$ and $\neg A$ being true.

\section{REFERENCES}

[1] B. Barshan and R. Kuc, "Differentiating sonar reflections from corners and planes by employing an intelligent sensor," IEEE Trans. Pattern Anal. Machine Intell., vol. 12, pp. 560-569, 1990.

[2] Borenstein and Y. Koren, "Obstacle avoidance with ultrasonic sensors," IEEE J. Robot. Automat., vol. 4, pp. 213-218, 1988.

[3] A. Elfes, "Sonar-based real-world mapping and navigation," IEEE J. Robot. Automat., vol. RA3, pp. 249-265, June 1987.

[4] J. J. Leonard, "Directed sonar sensing for mobile robot navigation," $\mathrm{Ph} . \mathrm{D}$. dissertation, University of Oxford, Oxford, U.K., 1991

[5] J. J. Leonard and H. F. Durrant-Whyte, "Dynamic map building for an autonomous mobile robot," Int. J. Robot. Res., vol. 11, Aug. 1992.

[6] J. H. Lim and D. W. Cho, "Physically based sensor modeling for sonar map in specular environment," in Proc. IEEE Int. Conf. Robot. Automat., 1992, pp. 1714-1719.

[7] L. Matthies and A. Elfes, "Integration of sonar and stereo range data using a grid-based representation," in Proc. IEEE Int. Conf. Robot. Automat., 1988, pp. 727-733.

[8] J. McKerrow, "Echolocation: From range to outline segments," Intelligent Autonomous Systems, F. C. Groen, S. Hirose, and C. E. Thorpe, Eds. Washington, DC: IOS, 1989, vol. IAS-3, pp. 238-247.

[9] P. J. McKerrow and J. C. T. Hallam, "An introduction to the physics of echolocation," in Proc. 3rd Nat. Conf. Robot., Australian Robot Association, Melbourne, Australia, June 1990, pp. 198-209.

[10] G. Shafer, A Mathematical Theory of Evidence. Princeton, NJ: Princeton Univ. Press, 1976.

[11] A. P. Tirumalai, B. G. Schunck, and R. J. Jain, "Evidential reasoning for building environment maps," IEEE Trans. Syst., Man Cybern., vol. 25, 1995.

[12] F. Gambino, G. Oriolo, and G. Ulivi, "A comparison of three uncertainty calculus techniques for ultrasonic map building," SPIE Int. Symp. Aerospace/Defense Sensing Contr., Orlando, FL, 1996, pp. 249-260.

\section{Sliding and Hopping Gaits for the Underactuated Acrobot}

\author{
Matthew D. Berkemeier and Ronald S. Fearing
}

Abstract-In this paper, a new example of a planar hopping robot is considered, which has only one actuated joint. Simulations demonstrate that the robot can perform both sliding and hopping gaits, despite the fact that almost all other hopping robots have at least two actuated joints.

Index Terms-Acrobot, double pendulum, hopping robot, legged robot.

\section{INTRODUCTION}

Most of the previous work on hopping in robotics has focused on the mechanism designed and built by Raibert [1]. His planar hopper had two actuated joints: a springy prismatic leg and a revolute hip joint. It was capable of hopping in place, hopping at various forward speeds, and leaping over small obstacles. The use of two actuated joints allowed Raibert to dedicate one actuator to controlling the hopping height and the other to controlling body attitude (note, however, that Raibert's robot was underactuated in the same sense as the robot studied in this paper). Others who have built hopping robots include Kanai and Yamafuji [2] and Papantoniou [3]. In both these cases as well, two actuated joints were used to achieve balance and control hopping height. In this paper, we consider the problem of controlling both balance and thrust with only one actuated joint.

Koditschek and Bühler, Vakakis et al., M'Closkey and Burdick, and $\mathrm{Li}$ and He [4]-[7] all studied the hopping cycle of Raibert's robot. By considering simplified dynamics of the hopper and the associated return maps, various predictions could be made about the hopper's behavior as a function of parameters. Among the interesting results were the existence of stable limit cycles with period 2 Poincarè maps (so-called "limping gaits"). Although this approach is, in principle, applicable to any legged robot, it would not be trivial to apply it the robot described in this paper. To derive a Poincarè map one needs to be able to solve the dynamical equations either exactly or approximately. The equations for the Acrobot are highly nonlinear and there is no closed-form exact solution. There is also not an obvious approximation method to apply to the Acrobot.

$\mathrm{Li}$ and Montgomery [8] studied the dynamics of the flight phase for Raibert's robot and considered the problem of optimally performing a somersault in the air by using the holonomy generated by internal motions. Although the goal in this paper is also to use internal motions to achieve a particular orientation while off the ground, no attempt is made to perform the motions in an optimal fashion. Others have examined control of legged robots in flight phase without a discussion of holonomy. Representative examples include Hodgins and Raibert [9], Playter and Raibert [10], and Lapshin [11].

Much was learned from studying Raibert's hopper. However, it is important to consider new examples. Typically, it is only by comparing the results of many specific examples of a phenomenon

Manuscript received March 25, 1997; revised April 1, 1998. This paper was recommended for publication by Associate Editor M. Hebert and Editor A. Goldenberg upon evaluation of the reviewers' comments.

M. D. Berkemeier is with the Department of Aerospace and Mechanical Engineering, Boston University, Boston, MA 02215 USA (e-mail: matthewb@gazelle.bu.edu).

R. S. Fearing is with the Department of Electrical Engineering and Computer Science, University of California, Berkeley, CA 94720-1770 USA (e-mail: ronf@robotics.eecs.berkeley.edu).

Publisher Item Identifier S 1042-296X(98)04518-2. 